\title{
Current fluctuations in a single tunnel junction
}

\author{
Hyunwoo Lee* \\ Department of Physics, Massachusetts Institute of Technology, 77 Massachusetts Ave., \\ Cambridge, MA 02139 \\ L. S. Levitovt \\ Department of Physics, Massachusetts Institute of Technology, 77 Massachusetts Ave., \\ Cambridge, MA 02139, \\ and Landau Institute for Theoretical Physics, 2 Kosygin St., Moscow 117334, Russia
}

\begin{abstract}
We study noise spectra of currents through a tunnel junction in weak tunneling limit. We introduce effective capacitance to take into account the interaction effect and explicitly incorporate the electromagnetic environment of the junction into the formulation. We study the effect of charging energy and macroscopic environment on noise spectra. We calculate current fluctuations at tunneling barrier and fluctuations measured at leads. It is shown that two fluctuations have different noise spectra and the relation between them is nontrivial. We provide an explanation for the origin of the difference. Experimental implications are discussed.
\end{abstract}

PACS numbers: 72.70.+m, 73.40.Gk, 73.40.Jn

Typeset using REVTEX 


\section{INTRODUCTION}

Even though "noise" usually represents unwanted fluctuations which blur information, noise is on the fundamental level closely connected with the dynamics of a system and it contains variety of information.

In this context, there have been many recent studies on a noise spectrum of current in small devices. In ballistic systems, the noise power can be suppressed to zero due to Pauli exclusion principle [1] 3]. In diffusive systems, the noise power is suppressed to $1 / 3$ of the classical value [ [4]. There have been attempts to study the effect of electron-electron interaction on the noise. For example, noise spectrum in 1D systems was calculated from Luttinger liquid approximation [5].6].

In this paper, we study current fluctuations of ultra-small tunnel junctions made of two pieces of metal separated by a thin insulating barrier.

It is helpful to discuss a few limiting cases. It is well known that conventional tunnel junctions show Ohmic behavior $I(V)=V / R_{T}$ when it is driven by external voltage bias and the current noise spectrum of Ohmic junctions follows Johnson-Nyquist formula [7]

$$
S_{I_{T}}(\omega)=\frac{1}{2 \pi R_{T}} \sum_{ \pm}(\hbar \omega \pm e V) \operatorname{coth}\left(\frac{\hbar \omega \pm e V}{2 k_{B} T}\right) .
$$

Recent advances in fabrication technology have made it possible to construct very small devices. It was pointed that electron-electron interaction effect becomes important for small devices. For example, it was argued that a tunnel junction develops tunneling gap in currentvoltage characteristic when the temperature is lower than the charging energy of a junction $E_{C}=e^{2} / 2 C[1]$.

The charging energy was predicted to affect noise spectrum as well. Ben-Jacob et al [13] studied fluctuations of current in an open tunnel junction. They calculated equilibrium noise of a junction and found that the low frequency noise $\hbar \omega<E_{C}$ is suppressed to zero in the low temperature limit $k_{B} T \ll E_{C}$.

Following theoretical predictions on the charging effect in a small tunnel junction, many 
experimental attempts were made to observe the charging effect or so called Coulomb blockade effect. Coulomb blockade effect was verified for multi-junction systems [14 20]. However the clear verification of the effect for a single tunnel junction has not been reported.

It was later realized that the electrical transport properties of a single tunnel junction crucially depends on the electromagnetic environment of a junction which is decided by properties of leads on junction chip [21 23]. Current-voltage characteristic was calculated with explicit account of the environment by modeling the environment as linear impedance presented to the junction. It was shown that the Coulomb blockade effect is erased by the quantum fluctuations of charge when the environment has low impedance and that high impedance environment is essential ingredient for the realization of Coulomb blockade effect. The dependence on the electromagnetic environment was experimentally observed [24]

Behaviors of single tunnel junctions are decided not only by the ratio of temperature and the charging energy but also by the environment. It is a goal of this paper to extend the earlier works to include noise spectrum calculation for general environment.

More important goal of this paper is to show that noise measurement in a single tunnel junction is quite nontrivial. For illustration, we calculate current fluctuations at the tunneling barrier and fluctuations at the leads.

Most theoretical works on noise calculated the current fluctuations at a specific point of a circuit, usually at a potential barrier right in the middle of a junction. However as pointed out by Landauer et al [25], conventional noise measurements have no relation with the electrons crossing the potential barrier of the junction. The noise is measured, for example, through the voltage across a series resistor in a circuit.

Noise measured in experiments is not precisely the same quantity as the noise treated in various theoretical calculations. Noise measured in experiments is complicated by the intrinsic noise of noise measuring device. To make comparison with theoretical calculations, it is usually assumed that the intrinsic noise makes linear contribution and that experiments can achieve agreement with theoretical calculations by subtracting off the intrinsic noise from the measured noise. We will call this linear superposition assumption [25,26]. 
In this paper, we assume that leads, which is connected to a junction and provides electromagnetic environment, can be used as a measuring device and calculate current fluctuations at leads. We also calculate current fluctuations at tunneling barrier and compare them with current fluctuations at leads to test the linear superposition assumption. To briefly mention a result of the calculation, we find that the linear superposition assumption holds only when

$$
|Z(\omega)| \ll \frac{1}{|i \omega C|},
$$

where $Z(\omega)$ is the impedance of the environment. In low impedance environment, the assumption breaks down due to finite relaxation time of environment. In high impedance environment, noise becomes quite nontrivial and spectral density at $\hbar \omega \approx \pm E_{C}$ can be reduced by applying small voltage bias. We will later provide the physical origin of this novel phenomena.

In Sec.II, we present a formulation of a problem which incorporates the charging energy and the environment. The formulation allows simple perturbation calculation in the weak tunneling limit. In Sec.III, we introduce two current operators. One corresponds to current at potential barrier of a junction, which we call tunneling current. The other corresponds to current measured at the leads, which we call relaxed current. Expectation values of the two operators are evaluated for a few examples of environment. In Sec.IV, the noise spectrum of the tunneling current is calculated. It shows agreement with existing theories in various limiting cases. In Sec. $\mathrm{V}$, the noise spectrum of the relaxed current is calculated. It is compared to the noise spectrum of the tunneling current and the difference is identified. The difference is attributed to the finite relaxation time of environment and nontrivial zero point fluctuations of environmental degree of freedom.

\section{FORMULATION}

Fig.1(a) presents a schematic diagram of a system which we consider in the paper. A junction is represented by a capacitor notation with an arrow right beside it to emphasize 
tunneling. The junction is connected to the electromagnetic environment and voltage source. It is assumed that the environment is linear so that it can be modeled as an impedance $Z(\omega)$. The impedance is determined by properties of leads connected to junction chip. For detailed discussion of environment and the model system in Fig.11(a), see Ref. [27].

We use the same formulation as the one adopted in Ref. [22]. The tunnel junction connected to the environment is described by the following hamiltonian(for more details about this hamiltonian, see [28),

$$
H=H_{q p}+H_{e n v}+H_{T} .
$$

The first term $H_{q p}$ describes quasi-particle degrees of freedom,

$$
H_{q p}=\sum_{k \sigma}\left(\epsilon_{k}+e V\right) c_{k \sigma}^{\dagger} c_{k \sigma}+\sum_{q \sigma} \epsilon_{q} c_{q \sigma}^{\dagger} c_{q \sigma}
$$

where $k$ and $q$ label the quasi-particle states in the left and the right electrodes, respectively and $\sigma$ represents spin degree of freedom.

The second term $H_{\text {env }}$ describes environmental degrees of freedom and the charging energy,

$$
H_{e n v}=\frac{\tilde{Q}^{2}}{2 C}+\sum_{n=1}^{N}\left[\frac{q_{n}^{2}}{2 C_{n}}+\left(\frac{\hbar}{e}\right)^{2} \frac{1}{2 L_{n}}\left(\tilde{\varphi}-\varphi_{n}\right)^{2}\right]
$$

where $\tilde{Q}$ is the charge on the capacitor plates of the junction with the steady state contribution subtracted off $\tilde{Q}=Q-C V$. $\tilde{\varphi}$ is the phase variable across the junction, $\tilde{\varphi}(t)=(e / \hbar) \int_{-\infty}^{t} d t^{\prime} \tilde{Q}\left(t^{\prime}\right) / C . \tilde{Q}$ and $\tilde{\varphi}$ are conjugate variables describing macroscopic state of the junction and they satisfy the quantization relation,

$$
[\tilde{\varphi}, \tilde{Q}]=i e
$$

Phase $\tilde{\varphi}$ is coupled to a set of harmonic oscillators following the Caldeira-Leggett description 29] of the environment.

The third term describes the tunneling between two plates of the capacitor,

$$
H_{T}=\sum_{k q \sigma} T_{k q} c_{q \sigma}^{\dagger} c_{k \sigma} e^{-i \tilde{\varphi}}+\text { H.c. }
$$


where $T_{k q}$ is the tunneling matrix element. It is simple to verify that $e^{-i \tilde{\varphi}}$ is a charge lowering operator,

$$
e^{i \tilde{\varphi}} \tilde{Q} e^{-i \tilde{\varphi}}=\tilde{Q}-e
$$

Because $e^{ \pm i \tilde{\varphi}}$ explicitly takes care of the charge increase and decrease, the quasi-particle degrees of freedom and the environment degrees of freedom can be separated so that quasiparticle operators commute with the charge and phase operators.

\section{TUNNELING CURRENT AND RELAXED CURRENT}

To calculate noise spectra, we need to construct current operators. The tunneling current operator can be easily identified from the operator equation of motion,

$$
\begin{aligned}
\hat{I}_{T} & =\frac{i}{\hbar}\left[H, e \sum_{q \sigma} c_{q \sigma}^{\dagger} c_{q \sigma}\right] \\
& =-\frac{e}{\hbar} \sum_{k q \sigma}\left(i T_{k q} c_{q \sigma}^{\dagger} c_{k \sigma} e^{-i \tilde{\varphi}}+\text { H.c. }\right) .
\end{aligned}
$$

This operator represents the net number of electrons tunneled from one plate of the capacitor

to the other. Experimentally, $\hat{I}_{T}$ corresponds to current measured right at the tunneling barrier(Fig.1(b)).

Eq.(9) is not a unique choice as a current operator and there is another way of constructing a current operator,

$$
\begin{aligned}
\hat{I}_{R} & =\frac{i}{\hbar}\left[H_{e n v}, \tilde{Q}\right] \\
& =-\frac{\hbar}{e} \sum_{n} \frac{1}{L_{n}}\left(\tilde{\varphi}-\varphi_{n}\right) .
\end{aligned}
$$

For definiteness, we will call $\hat{I}_{T}$ tunneling current and $\hat{I}_{R}$ relaxed current. These currents can be related in a simple way,

$$
\hat{I}_{R}-\hat{I}_{T}=\frac{i}{\hbar}[H, \tilde{Q}]=\frac{d \tilde{Q}}{d t},
$$

and from this relation, we interpret $\hat{I}_{R}$ as a charge flow at the environment. Fig.1 (1) illustrates the difference between $\hat{I}_{T}$ and $\hat{I}_{R}$. The construction of the relaxed current operator is possible because the environment is explicitly taken into account. 
Because we are interested in the weak tunneling limit, we take $H_{q p}+H_{e n v}$ as unperturbed hamiltonian and $H_{T}$ as perturbation. Both $H_{q p}$ and $H_{e n v}$ are quadratic and the perturbation calculation is straightforward.

Current-voltage characteristic can be obtained from the perturbation theory and one obtains

$$
I_{T}(V)=I_{R}(V)=\frac{2 e}{\hbar^{2}} \operatorname{Im}\left(X_{r e t}(-e V / \hbar)\right)
$$

where

$$
X_{r e t}(\omega)=-i \int_{-\infty}^{\infty} d t \Theta(t) e^{i \omega t}\left\langle\left[A(t) e^{-i \tilde{\varphi}(t)}, A^{\dagger}(0) e^{i \tilde{\varphi}(0)}\right]\right\rangle_{0}
$$

and $A(t)=\sum_{k q \sigma} T_{k q} c_{q \sigma}^{\dagger}(t) c_{k \sigma}(t) .\langle\cdots\rangle_{0}$ represents the average over unperturbed state and all operators are written in interaction picture. Because the expectation values of the tunneling current and the relaxed current are the same, we will drop the subscript. By explicitly evaluating the average, one obtains

$$
I(V)=\frac{1}{e R_{T}}\left(1-e^{-\beta e V}\right) \int_{-\infty}^{\infty} d E \frac{E}{1-e^{-\beta E}} P(e V-E)
$$

where

$$
P(E)=\frac{1}{2 \pi \hbar} \int_{-\infty}^{\infty} d t \exp \left[\langle[\tilde{\varphi}(t)-\tilde{\varphi}(0)] \tilde{\varphi}(0)\rangle_{0}+i E t / \hbar\right]
$$

Eq.(14) together with Eq.(15) agrees with the result of Ref. [22], which justifies two current operators.

All information of the environment is stored in the phase-phase correlation function $J(t)=\langle[\tilde{\varphi}(t)-\tilde{\varphi}(0)] \tilde{\varphi}(0)\rangle_{0}$ and from the fluctuation-dissipation theorem, $J(t)$ can be related to the impedance $Z(\omega)$,

$$
J(t)=2 \int_{0}^{\infty} \frac{d \omega}{\omega} \frac{\operatorname{Re} Z_{t}(\omega)}{R_{Q}}\left\{\operatorname{coth} \frac{\beta \hbar \omega}{2}[\cos \omega t-1]-i \sin \omega t\right\},
$$

where $Z_{t}(\omega)=\left(Z^{-1}(\omega)-i \omega C\right)^{-1}$ and $R_{Q}$ is the resistance quantum $h / e^{2}$.

Let us present current-voltage characteristics in a few special cases. It illustrates the effect of the environment and we later find that $S_{I_{T}}(\omega)$ is closely related to the characteristic. 
Firstly, let us take $Z(\omega)=0$. Then from Eq.(15, 16), one finds $P(E)=\delta(E)$ and the junction shows Ohmic behavior. The charging energy plays no role in the low impedance environment. Secondly, let us take $Z(\omega)=R \gg R_{Q}$ and $\beta E_{C} \gg 1$. Then from Eq.(15, 16), one finds $P(E)=\delta\left(E-E_{C}\right)$ and the characteristic develops a gap and current does not flow for $|e V|<E_{C}$. This agrees with the prediction of Likharev et al [11] and one realizes that their semiclassical theory becomes valid if a junction is connected to high impedance environment. Finally, let us take Ohmic environment of arbitrary resistance, $Z(\omega)=R$ [30]. In this case, the characteristic is rather complicated and we present only the limiting behaviors at zero temperature. With small voltage bias $|e V| \ll E_{C}$, one finds [22]

$$
I(V)=\frac{\exp \left(-2 \gamma R / R_{Q}\right)}{\Gamma\left(2+2 R / R_{Q}\right)} \frac{V}{R_{T}}\left[\frac{\pi R}{R_{Q}} \frac{|e V|}{E_{C}}\right]^{2 R / R_{Q}},
$$

where $\gamma=0.577 \ldots$ is the Euler constant. Note that Eq.(17) smoothly interpolates the characteristics of the low and the high impedance environments. Also note that with the finite impedance, the suppression due to Coulomb blockade effect is not strong enough to develop well defined gap even at zero temperature. With large voltage bias $e V \gg E_{C}$, one finds [28] the characteristic approaches the high impedance result,

$$
I(V)=\frac{1}{e R_{T}}\left[e V-E_{C}+\frac{R_{Q}}{\pi^{2} R} \frac{E_{C}^{2}}{e V}\right] .
$$

The zero temperature current-voltage characteristics are presented in Fig.2. For the experimental measurement of the current-voltage characteristic, see Ref. [24].

\section{NOISE SPECTRUM OF TUNNELING CURRENT}

\section{A. Noise spectrum of tunneling current}

Noise spectral density of current or noise spectrum is defined as Fourier transform of a current-current correlation function,

$$
S_{I}(\omega)=\frac{1}{\pi} \int d t e^{i \omega t}\left(\frac{1}{2}\langle\{\hat{I}(t), \hat{I}(0)\}\rangle-\langle\hat{I}(t)\rangle\langle\hat{I}(0)\rangle\right) .
$$


Because we later find the noise spectrum of the tunneling current different from the one of the relaxed current, we will put subscript $T$ and $R$ to give distinction.

Calculation of $S_{I_{T}}(\omega)$ is as straightforward as the calculation of the current-voltage characteristic and one finds

$$
\begin{aligned}
S_{I_{T}}(\omega)= & \frac{1}{2 \pi R_{T}} \int d E \frac{E}{1-e^{-\beta E}} \\
& \times \sum_{ \pm} P(\hbar \omega \pm e V-E)\left(1+e^{-\beta(\hbar \omega \pm e V)}\right) .
\end{aligned}
$$

Structure of Eq. (20) is similar to the formula for the current-voltage characteristic Eq.(14) and indeed, $S_{I_{T}}(\omega)$ can be written in terms of $I(V)$,

$$
S_{I_{T}}(\omega)=\frac{e}{2 \pi} \sum_{ \pm} \operatorname{coth} \frac{\beta(e V \pm \hbar \omega)}{2} I(V \pm \hbar \omega / e) .
$$

Eq.(21) allows one to obtain the noise spectrum of the tunneling current from the currentvoltage characteristic.

It is a simple task to verify that Eq.(21) satisfies the fluctuation-dissipation theorem in equilibrium $V=0$. To verify it, one introduces a coupling of the tunneling current to its conjugate force $f_{T}(t)$,

$$
H_{f_{T}}=-\hat{I}_{T} f_{T}(t)
$$

and calculates the response function $\chi_{T}(\omega)=\left\langle\hat{I}_{T}(t)\right\rangle_{\omega} / f_{T}(\omega)$. Then, the fluctuationdissipation theorem relates the response to the noise spectrum

$$
S_{I_{T}}(\omega)=\hbar \operatorname{coth} \frac{\beta \hbar \omega}{2} \operatorname{Im} \chi_{T}(\omega) .
$$

In the following, we consider equilibrium and excess noise for Ohmic environment at zero temperature. Finite temperature noise will be discussed only when it allows a simple analytic expression.

\section{B. Low impedance environment}

Let us first study the low impedance limit $Z(\omega)=R \ll R_{Q}$, where the junction becomes Ohmic. In equilibrium, Eq.(21) reduces to Johnson-Nyquist noise [31] (Fig.3) 


$$
S_{I_{T}}(\omega)=\frac{1}{\pi R_{T}} \hbar \omega \operatorname{coth} \frac{\beta \hbar \omega}{2} .
$$

Excess noise is the difference between equilibrium and non-equilibrium noise $S_{I_{T}}^{X}(\omega ; V)=$ $S_{I_{T}}(\omega ; V)-S_{I_{T}}(\omega ; V=0)$. The superscript $X$ is employed to denote the excess noise.

At zero temperature, the excess noise is absent for $|\hbar \omega|>|e V|$ and for $|\hbar \omega|<|e V|$, it shows piecewise linear dependence on frequency (Fig.|⿴囗大)(a)),

$$
S_{I_{T}}^{X}(\omega ; V)=\frac{|e V|-|\hbar \omega|}{\pi R_{T}}
$$

Eq.(24) and Eq.(25) agree with Eq.(11) where the charging effect was neglected. The agreement with the previous study illustrates that the charging effect does not show up in the low impedance environment.

\section{High impedance environment}

With $Z(\omega)=R \gg R_{Q}$, Eq.(21) reduces to

$$
S_{I_{T}}(\omega)=\frac{1}{\pi R_{T}}\left(1+e^{-\beta \hbar \omega}\right) \int_{-\infty}^{\infty} d E \frac{E}{1-e^{-\beta E}} P(\hbar \omega-E),
$$

in equilibrium where

$$
P(E)=\frac{1}{\sqrt{4 \pi E_{C} k_{B} T}} \exp \left[-\frac{\left(E-E_{C}\right)^{2}}{4 E_{C} k_{B} T}\right] .
$$

At high temperature $k_{B} T \gg E_{C}, S_{I_{T}}(\omega)$ becomes $\left(2 k_{B} T / \pi R_{T}\right)\left(1-E_{C} / 3 k_{B} T+\mathcal{O}\left(E_{C} / k_{B} T\right)^{2}\right)$ for $\hbar \omega \ll E_{C}$, which is the Coulomb blockade correction to white noise. At low temperature $k_{B} T \ll E_{C}, S_{I_{T}}(\omega)$ becomes $\left(2 E_{C} / \pi R_{T}\right) \exp \left(-E_{C} / k_{B} T\right)$ for $\hbar \omega \ll k_{B} T$. The Coulomb

blockade strongly suppresses the noise far below the white noise level in the low temperature (Fig.局).

Both high and low temperature limiting behaviors agree with earlier calculation of BenJacob et al [13] where they calculated the equilibrium noise spectrum of an open junction which is not connected to the external circuit. Ben-Jacob et al's result can be cast into the form of Eq.(26) with $P(E)$ replaced by 


$$
P_{B J}(E)=\left[\sum_{\text {integer }_{q}} \exp \left(-\frac{q^{2} E_{C}}{k_{B} T}\right)\right]^{-1} \sum_{q} \delta\left(E-E_{C}(2 q+1)\right) \exp \left(-\frac{q^{2} E_{C}}{k_{B} T}\right) .
$$

Note that $P_{B J}(E)$ is a discrete form of $P(E) . P(E)$ and $P_{B J}(E)$ become identical in the high temperature limit $k_{B} T \gg E_{C}$ whereas they exhibit delicate difference in the low temperature limit $k_{B} T \ll E_{C}$. Recalling that $P(E)$ is a probability to emit energy $E$ to environment [28], one finds that Eq.(28) implies the quantization of the emitted energy whereas Eq.(27) contains continuous spectrum of the emitted energy.

The charge in each electrode is quantized both in the open junction and in the closed junction with high impedance environment. Hence the energy used to charge the electrodes has only discrete values. In the open junction, the whole emitted energy is used to charge the electrodes. In the junction closed by the environment, however, the emitted energy is used only in part to charge the electrodes and the rest excites the environmental degrees of freedom. Therefore $P(E)$ has continuous spectrum of energy whereas $P_{B J}(E)$ allows only discrete energy values.

In the high impedance environment, the excess noise shows piecewise linear dependence on frequency at zero temperature(Fig. $4(\mathrm{a}, \mathrm{b}))$. For small voltage bias $|e V|<E_{C}$, the excess noise has finite spectral density(Fig. $4(\mathrm{a})$ ),

$$
S_{I_{T}}^{X}(\omega)=\frac{|e V|-|| \hbar \omega\left|-E_{C}\right|}{2 \pi R_{T}},
$$

for $-|e V|<|\hbar \omega|-E_{C}<|e V|$. Note that even though current does not flow when $|e V|<E_{C}$, the excess noise does not vanish around $\hbar \omega= \pm E_{C}$. At higher voltage bias, finite current flows and Fig. $1(\mathrm{~b})$ shows the evolution of the excess noise with the voltage. The excess noise again shows the piecewise linear dependence. The piecewise linear dependence in both the low and the high impedance environments originate from the narrow energy range of $P(E)$.

\section{Ohmic impedance environment}

One can also obtain $S_{I_{T}}(\omega)$ in arbitrary Ohmic impedance environment $Z(\omega)=R$. In the low frequency regime $|\hbar \omega| \ll E_{C}$, the zero temperature equilibrium fluctuations are 
suppressed by the Coulomb blockade effect,

$$
S_{I_{T}}(\omega)=\frac{\exp \left(-2 \gamma R / R_{Q}\right)}{\pi \Gamma\left(2+2 R / R_{Q}\right)} \frac{|\hbar \omega|}{R_{T}}\left[\frac{\pi R}{R_{Q}} \frac{|\hbar \omega|}{E_{C}}\right]^{2 R / R_{Q}} .
$$

However the suppression is not complete even at zero temperature. The degree of the suppression depends on the impedance and the higher the impedance, the stronger the suppression is.

In the high frequency regime $|\hbar \omega| \gg E_{C}$, the equilibrium noise eventually converges to the result of the high impedance environment,

$$
S_{I_{T}}(\omega)=\frac{e}{\pi R_{T}}\left[|\hbar \omega|-E_{C}+\frac{R_{Q}}{\pi^{2} R} \frac{E_{C}^{2}}{|\hbar \omega|}\right],
$$

and the convergence is faster with the higher impedance (Fig.3).

With finite Ohmic impedance, the excess noise no longer exhibits piecewise linear dependence on the frequency because $P(E)$ allows broad range of energy. Since the Coulomb blockade effect is blurred by the quantum fluctuations of charge in the finite impedance environment, the excess noise is finite in broad frequency range (Fig. (4)(a)). At zero frequency,

$$
S_{I_{T}}^{X}(\omega=0)=\frac{1}{\pi} \frac{\exp \left(-2 \gamma R / R_{Q}\right)}{\Gamma\left(2+2 R / R_{Q}\right)} \frac{|e V|}{R_{T}}\left[\frac{\pi R}{R_{Q}} \frac{|e V|}{E_{C}}\right]^{2 R / R_{Q}} .
$$

\section{E. Noise power}

Before we close the discussion of the tunneling current noise spectrum, let us mention one implication of Eq.(21). In the presence of finite current, the correlation between charge tunneling events can be estimated from the noise power $P$, which is defined as $P=2 \pi S_{I}(\omega=$ $0)$. If there is no correlation between the tunneling events, the noise power retains its full value $P=2 e I$. If there is any kind of correlation which regulates tunneling, it decrease the noise power below its full value. For example, in a single channel quantum point contact, the Fermi correlation is known to reduce the noise power to $P=2 e I(1-\mathcal{T})$ where $\mathcal{T}$ is the transmission coefficient [1] 3 ]. 
From Eq.(21), one finds the noise power of the tunneling current to have the full value independent of the environment,

$$
P_{T}=2 e I(V)
$$

where the subscript $T$ is employed to denote that this is the noise power of the tunneling current. The full noise power implies that Fermi statistics and the charging energy do not give rise to any correlation between tunneling events. This result is understandable since we are studying the weak tunneling limit and the time interval between two consecutive tunneling events is assumed to be large.

\section{NOISE SPECTRUM OF RELAXED CURRENT}

\section{A. Noise spectrum of relaxed current}

Most theoretical works on current fluctuations calculates the noise spectrum from electron crossing of a particular plane in a circuit. However, as Landauer and Martin state [25] 'Currents are measured either through the voltage developed across a small series resistor in the circuit, or through the magnetic fields generated by the displacement current. Contrary to occasional perceptions, noise measurements have no relation to the crossing, by carriers, of a particular plane along the circuit.'

To clarify the relation between conventional noise measurements and noise spectrum treated in various theoretical works [1, 3, 7, 13, 32, it is necessary to take an explicit account of a measuring device in the formulation. We assume that leads connected to a junction can be used as a measuring device and calculate current fluctuations at leads. The relaxed

current $\hat{I}_{R}$ corresponds to charge flow at leads and therefore $S_{I_{R}}(\omega)$ corresponds to spectral density of current fluctuations measured at leads. We will study the difference between $S_{I_{R}}(\omega)$ and $S_{I_{T}}(\omega)$ in this section.

No tunneling limit $R_{T} \rightarrow \infty$ illustrates a crucial difference of $S_{I_{R}}(\omega)$ and $S_{I_{T}}(\omega)$. In this limit, the tunneling current operator itself vanishes because it is proportional to the 
tunneling matrix elements. Hence, one finds $S_{I_{T}}(\omega)=0$. This result is natural since $\hat{I}_{T}$ is the electron flow measured right at the tunneling barrier and electrons cannot tunnel the barrier at all in the no tunneling limit. On the other hand, the relaxed current operator does not depend on the tunneling matrix elements explicitly and in the zero tunneling limit, one finds $S_{I_{R}}(\omega)=S_{I_{R}}^{(0)}(\omega)$ where

$$
S_{I_{R}}^{(0)}(\omega)=\frac{1}{\pi} \operatorname{Re}\left(\frac{1}{Z(\omega)-\frac{1}{i \omega C}}\right) \hbar \omega \operatorname{coth} \frac{\beta \hbar \omega}{2} .
$$

The superscript (0) denotes that this is the zeroth order contribution in the perturbation calculation. Note that $S_{I_{R}}^{(0)}(\omega)$ is a generalization of Johnson-Nyquist noise [31] $S_{I}(\omega)=$ $1 /(\pi R) \hbar \omega \operatorname{coth}(\beta \hbar \omega / 2)$. $\quad S_{I_{R}}(\omega)$ measures the fluctuations of the relaxed current in the environment and $S_{I_{R}}(\omega)$ does not vanish even in the no tunneling limit because of the zero point fluctuations in the environment.

With finite tunneling, it is usually assumed that $S_{I_{R}}(\omega)$ is a linear superposition of the intrinsic noise $S_{I_{R}}^{(0)}(\omega)$ and the tunneling noise $S_{I_{T}}(\omega)$, which we call linear superposition assumption. The assumption is frequently used to make comparison of experiments with theoretical calculations (for example, see Ref. [26]).

Explicit calculation of $S_{I_{R}}(\omega)$ to the second order in the tunneling matrix produces

$$
\begin{aligned}
& S_{I_{R}}(\omega)=S_{I_{R}}^{(0)}(\omega)+S_{I_{R}}^{(2)}(\omega) \\
& S_{I_{R}}^{(2)}(\omega)=S_{I_{R}}^{(2 A)}(\omega)+S_{I_{R}}^{(2 B)}(\omega)+S_{I_{R}}^{(2 C)}(\omega),
\end{aligned}
$$

where

$$
\begin{aligned}
S_{I_{R}}^{(2 A)}(\omega)= & \frac{1}{|1-i \omega C Z(\omega)|^{2}} S_{I_{T}}(\omega), \\
S_{I_{R}}^{(2 B)}(\omega)= & -\frac{e}{\pi}\left\{\operatorname{Im} \frac{1}{1-i \omega C Z(\omega)}\right\}^{2} \operatorname{coth} \frac{\beta \hbar \omega}{2} \sum_{ \pm} \pm I(V \pm \hbar \omega / e) \\
S_{I_{R}}^{(2 C)}(\omega)= & \frac{e^{2}}{\pi \hbar^{2}} \operatorname{Im}\left(\frac{1}{\{1-i \omega C Z(\omega)\}^{2}}\right) \operatorname{coth} \frac{\beta \hbar \omega}{2} \\
& \times \operatorname{Re} \sum_{ \pm}\left\{X_{\text {ret }}(-e V / \hbar \pm \omega)-X_{\text {ret }}(-e V / \hbar)\right\} .
\end{aligned}
$$

The superscript (2) in $S_{I_{R}}^{(2)}(\omega)$ denotes that this is the second order contribution in the perturbation calculation. Unlike the intrinsic noise, $S_{I_{R}}^{(2)}(\omega)$ depends on the voltage. 
At zero frequency, one finds $S_{I_{R}}^{(2 A)}(0)=S_{I_{T}}(0)$ and $S_{I_{R}}^{(2 B)}(0)=S_{I_{R}}^{(2 C)}(0)=0$, so that that the linear superposition assumption holds at zero frequency for arbitrary environment. Because $S_{I_{R}}^{(0)}(\omega)$ vanishes at zero frequency, one also finds that noise powers of the tunneling current and the relaxed current are always the same $P_{R}=P_{T}$. For non-zero frequency, one finds that the assumption holds if

$$
|Z(\omega)| \ll \frac{1}{|i \omega C|},
$$

and it does not otherwise. Eq.(37) has interesting implications. For example, in the Ohmic environment with high impedance $Z(\omega)=R \gg R_{Q}$ where Coulomb blockade effect is clear, the excess noise of the tunneling current exhibits interesting feature at frequencies around $\hbar \omega= \pm E_{C}$. However Eq. (37) predicts that at this frequency range, the excess noise of the relaxed current is noticeably different from the tunneling excess noise since Eq.(37) translates to $|\hbar \omega| \ll\left(R_{Q} / R\right) E_{C}($ see Sec. $\nabla \mathrm{Q})$.

Let us first discuss the equilibrium noise. Due to the fluctuation-dissipation theorem, the equilibrium noise spectrum can be alternatively derived by coupling the relaxed current to its conjugate force

$$
H_{f_{R}}=-\hat{I}_{R} f_{R}(t)
$$

and calculating the response function. This procedure produces

$$
\begin{aligned}
S_{I_{R}}(\omega)= & S_{I_{R}}^{(0)}(\omega)-\frac{e^{2}}{\pi \hbar^{2}} \operatorname{coth} \frac{\beta \hbar \omega}{2} \operatorname{Im}\left\{\frac{1}{(1-i \omega C Z(\omega))^{2}}\right. \\
& \left.\times\left(X_{\text {ret }}(\omega)+X_{\text {ret }}^{*}(-\omega)+-X_{\text {ret }}(0)-X_{\text {ret }}^{*}(0)\right)\right\} .
\end{aligned}
$$

It can be verified that Eq.(39) agrees with Eq.(35) in equilibrium. Note that though both $S_{I_{R}}(\omega)$ and $S_{I_{T}}(\omega)$ satisfy the fluctuation-dissipation theorem, they are still different since they are related to different response functions.

\section{B. Low impedance environment}

To make predictions from Eq.(35), one has to evaluate the real part of the retarded Green's function $X_{r e t}(\omega)$. For uniform tunneling density of states, one finds at zero temper- 
ature

$$
\begin{aligned}
& \operatorname{Re} \sum_{ \pm}\left\{X_{r e t}(-e V / \hbar \pm \omega)-X_{\text {ret }}(-e V / \hbar)\right\} \\
= & \frac{\hbar^{2}}{2 \pi e^{2} R_{T}} \int d E(P(E)-P(-E)) \\
& \times \sum_{ \pm}\{(e V-E \pm \hbar \omega) \ln |e V-E \pm \hbar \omega|-(e V-E) \ln |e V-E|\} .
\end{aligned}
$$

In the low impedance environment $Z(\omega)=R \ll R_{Q}$, one finds $S_{I_{R}}^{(2 C)}(\omega)$ vanishes at zero temperature and the zero temperature equilibrium noise becomes

$$
S_{I_{R}}(\omega)=S_{I_{R}}^{(0)}(\omega)+\frac{1}{\pi R_{T}} \operatorname{Re}\left(\frac{1}{(1-i \omega C Z(\omega))^{2}}\right)|\hbar \omega| .
$$

It can be verified that Eq.(41) agrees, to the leading order in $1 / R_{T}$, with zero temperature equilibrium noise in the presence of a shunt resistor $R_{T}$ in parallel with a capacitor $C$

$$
S_{I}(\omega)=\frac{1}{\pi} \operatorname{Re}\left(\frac{1}{Z(\omega)+\frac{1}{-i \omega C+1 / R_{T}}}\right)|\hbar \omega| .
$$

When the voltage bias is applied, one finds the zero temperature excess noise

$$
S_{I_{R}}^{X}(\omega)=\frac{1}{1+\omega^{2} R^{2} C^{2}} S_{I_{T}}^{X}(\omega)
$$

Eq.(43) can be understood if we assume that current fluctuations induced by tunneling is incoherent with equilibrium zero point current.

Then the behavior of a junction is essentially classical. The only effect of the tunneling is to introduce additional charge to plates of capacitor and from a conventional circuit analysis of a capacitor connected to impedance $Z(\omega)$ [33], one finds the current induced by tunneling is 28

$$
I_{R}(t)=\int d s \mathcal{R}(t-s) I_{T}(s)
$$

where $\mathcal{R}(t)=0$ for $t<0$ and $\mathcal{R}(\omega)=1 /(1-i \omega C Z(\omega))$. From the incoherence assumption, one immediately finds

$$
S_{I_{R}}^{X}(\omega)=\frac{1}{|1-i \omega C Z(\omega)|^{2}} S_{I_{T}}^{X}(\omega)
$$


which agrees with Eq.(43).

The factor $1 /|1-i \omega C Z(\omega)|^{2}$ in Eq. (45) originates from the finite relaxation time of the environment; the relaxation of tunneled charge to the environment is not immediate and it takes characteristic time of $C Z(\omega)$.

Fig.5(a) shows the relaxed excess noise in comparison to the tunneling excess noise. At zero temperature, there are three relevant energy scales $e V, \hbar / R C, E_{C}$. Fig.5(a) assumes $e V \gg \hbar / R C \gg E_{C}$. Due to the low impedance of the environment, the junction shows Ohmic behavior. The tunneling excess noise(dashed line) decays linearly with the frequency and its peak width is $\mathrm{eV}$. The relaxed excess noise(solid line) has the same magnitude with the tunneling excess noise at zero frequency. However, its peak width is given as $\min (e V, \hbar / R C)$ and for the case considered in Fig.5(a), it is $\hbar / R C$, making the decay of the relaxed excess noise faster than the tunneling excess noise.

\section{High impedance environment}

In the high impedance environment, the junction becomes highly nonlinear and the shunt resistor model cannot explain its behavior. To understand the junction behavior in the high impedance environment, the investigation of the zero point fluctuations in the environment is needed.

In the no tunneling limit, the environment is decoupled from quasi-particle degrees of freedom. The environmental hamiltonian is quadratic and therefore it can be diagonalized as a set of independent harmonic oscillators. Each harmonic oscillator shows zero point fluctuations of the magnitude $\left\langle x^{2}\right\rangle=\hbar /\left(2 m_{0} \omega_{0}\right) \operatorname{coth} \beta \hbar \omega_{0} / 2$ where $m_{0}$ and $\omega_{0}$ are mass and frequency of the oscillator, respectively. Harmonic oscillator with frequency $\omega_{0}$ contribute to

the spectral density of the relaxed current at $\omega=\omega_{0}$ and the zeroth order spectral density $S_{I_{R}}^{(0)}(\omega)$ is a sum of such contributions.

The situation becomes complicated if one turn on tunneling. The tunneling couples each harmonic oscillator to quasi-particle degrees of freedom and other harmonic oscillators. 
Therefore the tunneling provides each harmonic oscillator with the coupling to an effective heat reservoir which consists of other harmonic oscillators and quasi-particle degrees of freedom.

The effect of such coupling is twofold. It provides a noise source and it also introduces dissipation. The noise source tends to increase fluctuations in, for example, harmonic oscillator displacement variables while the dissipation has the opposite effect. It is known (for example, see Ref. [34]) that the coupling to the reservoir can even reduce the zero point point fluctuations in certain situations.

The relaxed current $\hat{I}_{R}$ is linear in the environmental variables. Therefore it can be written as a linear combination of harmonic oscillator displacements and the fluctuations of $\hat{I}_{R}$ is directly related to the fluctuations of harmonic oscillators.

A harmonic oscillator coupled to the reservoir is a difficult problem in general. However in the weak tunneling limit, one can perturbatively calculate the effect of the reservoir.

It is worth mentioning that the voltage bias is a variable describing quasi-particle degrees of freedom. Therefore fluctuations of harmonic oscillators and the relaxed current depend on the voltage bias.

Fig. (b) shows the relaxed excess noise in comparison to the tunneling excess noise at zero temperature in the high impedance Ohmic environment $Z(\omega)=R \gg R_{Q}$. The relaxed excess noise can be interpreted as change of harmonic oscillator fluctuations by the voltage bias. It is assumed that $E_{C}>e V \gg \hbar / R C$. Note that current does not flow because the voltage bias is below the gap $\mathrm{eV}<E_{C}$. The tunneling excess noise shows two peaks at $\hbar \omega= \pm E_{C}$. In comparison, the relaxed excess noise(solid line) becomes negative at $\hbar \omega \approx \pm E_{C}$. The relaxed excess noise is multiplied by $10^{4}$ to magnify the negative dips. Though the dips are quite shallow, it is important to notice that the dips imply the decrease of the total noise upon the application of the voltage bias. The dash-dotted line shows $1 /\left(1+\omega^{2} R^{2} C^{2}\right) S_{I_{R}}^{X}(\omega)$. The dash-dotted line is also multiplied by $10^{4}$. At higher voltage bias $e V>E_{C}$, the relaxed excess noise shows a peak at $\hbar \omega=0$ and shallow dips at $\hbar \omega \approx \pm E_{C}$. 
From Fig.5(b) and the relation $S_{I_{R}}^{X}(0)=S_{I_{T}}^{X}(0)$, one finds that the dissipative effect is dominant at $\hbar \omega \approx \pm E_{C}$ while the noise source effect is dominant at $\hbar \omega \approx 0$.

\section{Ohmic impedance environment}

Fig.5(c) shows the two excess noises at zero temperature in the Ohmic environment $Z(\omega)=R$. It is assumed that $R=R_{Q}$ and $e V=0.5 E_{C}$. The tunneling excess noise(dashed line) shows two peaks, while the relaxed excess noise(solid line) shows a single peak at $\hbar \omega=0$. The dash-dotted line shows $1 /\left(1+\omega^{2} R^{2} C^{2}\right) S_{I_{T}}^{X}(\omega)$ for comparison. The dashdotted line exhibit qualitatively the same feature as $S_{I_{R}}(\omega)$ though there are quantitative difference.

\section{E. Experimental implications}

Eq.(35) makes one interesting prediction in the low impedance Ohmic environment. As illustrated in Sec.VB (Fig.5(a)), the excess noise measured from the environment is different from the tunneling excess noise and follows Eq.(43).

Recently, there was an experiment which measured the excess noise of the quantum point contact in the low impedance environment [35]. The voltage bias $V$ of the order of $1 \mathrm{mV}$ was applied and the excess noise was measured at frequency $\omega \sim 10 \mathrm{GHz}$ which is much lower than the voltage $\hbar \omega \ll e V$. The frequency was chosen to avoid complications due to other noise sources at low frequency such as $1 / f$ noise. The measured excess noise was compared with theoretical calculations of the noise power(for example, Ref. [32] or $S_{I_{T}}^{X}(\omega)$ ) based on the assumption that the non-zero frequency excess noise is essentially the same as the noise power if $\hbar \omega \ll e V$. The experiment showed nice agreement with existing theories in ballistic regime. However in the pinched off regime which corresponds to the weak tunneling limit, it was found that the noise was reduced to about $1 / 3$ of theoretical predictions.

The quantum point contact in Ref. [35] is induced electrostatistically in the plane of a 2DEG embedded in GaAs-AlGaAs heterostructure. Due to the very different density of 
charge carriers in metals and semiconductors, our calculation on metallic junctions cannot be directly applied to analyze the experiment. However we believe that the difference of $S_{I_{R}}^{X}(\omega)$ and $S_{I_{T}}^{X}(\omega)$ in the low impedance environment is a generic property of the weak tunneling limit because the difference originates from the finite relaxation time of the environment which does not depend on charge carrier density.

We argue that the noise suppression observed in Ref. 35 may have its origin in the different frequency dependence of the relaxed and tunneling excess noise as illustrated in Sec.VB. However, we are unable to establish quantitative agreement of the experiment with Eq.(43) because the precise values of $R$ and $C$ are not available. More experiment on, for example, the frequency dependence of the suppression factor, is needed.

Eq.(35) makes another interesting prediction in the high impedance Ohmic environment. At frequency $\hbar \omega \approx \pm E_{C}$, the spectral density $S_{I_{R}}(\omega)$ measured at the leads decreases below its equilibrium value when the voltage bias is applied(Sec. $\nabla \mathrm{Q})$, making the relaxed excess noise negative. Usually the spectral density increases when the voltage bias is applied. Therefore the observation of the negative excess noise can serve as a crucial test of the present calculation.

\section{F. Weak scattering limit}

Calculation in this paper is restricted to the weak tunneling limit $R_{T} \gg R_{Q}$. It will be interesting to carry out similar study for the weak scattering limit. According to a recent experiment on quantum point contact [35], the measured excess noises show the deviation from existing theories only in the pinched off regime and as a channel opens(as a transmission coefficient $\mathcal{T}$ approaches 1 ), the deviation disappears. If the deviation is due to the difference between the relaxed excess noise and the tunneling excess noise, the experiment implies that the junction in the weak scattering limit no longer behaves as a conventional capacitor and the difference between the two excess noise vanishes. Though the experiment used junctions in semiconducting heterostructure, we expect metallic junctions show similar behavior at 
least in the low impedance environment.

Also there was a theoretical study of a single tunnel junction using the functional integral technique [36]. It was claimed that the junction becomes purely Ohmic and the capacitive effect vanishes in the weak scattering limit.

In the weak scattering limit, electron transport is continuous rather than being sporadic as in the weak tunneling limit and we don't expect a conventional capacitor is a proper description of the junction. However, further study is required to gain the quantitative understanding in the weak scattering limit.

\section{CONCLUSION}

In summary, we considered current transport through a junction connected to electromagnetic environment in the weak tunneling limit. The charging energy is taken into account to incorporate the electron-electron interaction effect. We calculated both the tunneling noise and the relaxed noise for arbitrary environment and showed their dependence on the charging energy and environment. We found that contrary to conventional expectation, the current through the potential barrier of the junction and the current measured from the environment have different excess noise. In the low impedance environment, the difference is due to the finite relaxation time of the junction. In the high impedance environment, additional complication occurs and at frequency $\hbar \omega \approx \pm E_{C}$, the spectral density of the relaxed current decreases below the equilibrium level when small voltage bias is applied.

\section{ACKNOWLEDGMENTS}

We are grateful to M. Reznikov for kindly sending us their manuscript before publication. Research of L.L is partly supported by Alfred Sloan Fellowship. 


\section{REFERENCES}

* email: HWLEE@MIT.EDU .

$\dagger \quad$ email: LEVITOV@MIT.EDU .

[1] G. B. Lesovik, Pis'ma Zh. Eksp. Teor. Fiz. 49, 513 (1989). (JETP Lett. 49, 592 (1989)).

[2] B. Yurke and G. P. Kochanski, Phys. Rev. B 41, 8184 (1990).

[3] M. Büttiker, Phys. Rev. Lett. 65, 2901 (1990).

[4] C. W. J. Beenakker and M. Müttiker, Phys. Rev. B 46, 1889 (1992).

[5] C. de C. Chamon, D. E. Freed, and X. G. Wen, Phys. Rev. B51, 2363 (1995).

[6] P. Fendley, A. W. W. Ludwig, and H. Saleur, cond-mat/9505031.

[7] A. J. Dahm, A. Denenstein, D. N. Langenberg, W. H. Parker, D. Rogovin, and D. J. Scalapino, Phys. Rev. Lett. 22, 1416 (1969).

[8] K. K. Likharev and A. B. Zorin, J. Low. Temp. Phys. 59, 347 (1985).

[9] D. V. Averin and K. K. Likharev, J. Low Temp. Phys. 62, 345 (1986).

[10] U. Geigenmüller and G. Schön, Physica (Amsterdam) 152B, 186 (1988).

[11] K. K. Likharev, IBM J. Res. Dev. bf 32, 144 (1988).

[12] D. V. Averin and K. K. Likharev, in: Mesoscopic Phenomena in Solids, ed. by B. L. Al'tshuler, P. A. Lee, and R. A. Webb (Elsevier, Amsterdam, 1991), Chap. 6.

[13] E. Ben-Jacob, E. Mottola, and Gerd Schön, Phys. Rev. Lett. 51, 2064 (1983).

[14] J. Lambe and R. C. Jaklevic, Phys. Rev. Lett. 22, 1371 (1969).

[15] T. A. Fulton and G. J. Dolan, Phys. Rev. Lett. 59, 109 (1987).

[16] P. J. M. van Bentum, R. T. M. Smokers, and H. van Kempen, Phys. Rev. Lett. 60, 2543 (1988). 
[17] L. S. Kuzmin, P. Delsing, T. Claeson, and K. K. Likharev, Phys. Rev. Lett. 62, 2539 (1989).

[18] R. Wilkins, E. Ben-Jacob, and R. C. Jaklevic, Phys. Rev. Lett. 63, 801 (1989).

[19] P. Delsing, K. K. Likharev, L. S. Kuzmin, and T. Claeson, Phys. Rev. Lett. 63, 1180 (1989).

[20] L. J. Geerligs, V. F. Anderegg, C. A. van der Jeugd, J. Romijin, and J. E. Mooij, Europhys. Lett. 10, 79 (1989).

[21] Yu. V. Nazarov, Pis'ma Zh. Eksp. Teor. Fiz. 49, 105 (1989) [ JETP Lett. 49, 126 (1989) ].

[22] M. H. Devoret, D. Esteve, H. Grabert, G. -L. Ingold, H. Pothier, and C. Urbina, Phys. Rev. Lett. 64, 1824 (1990).

[23] S. M. Girvin, L. I. Glazman, M. Jonson, D. R. Penn, M. D. Stiles, Phys. Rev. Lett. 64, 3183 (1990).

[24] A. N. Cleland, J. M. Schmidt, and J. Clarke, Phys. Rev. Lett. 64, 1565 (1990).

[25] R. Landauer and Th. Martin, Physica B175, 167 (1991).

[26] F. Liefrink, J. I. Dijkhuis, M. J. M. de Jong, L. W. Molenkamp, and H. van Houten, Phys. Rev. B 49, 14066 (1994).

[27] M. H. Devoret and H. Grabert, in: Single Charge Tunneling, ed. by A. Grabert and M. Devoret (Plenum, New York, 1992), Chap. 1.

[28] G. -L. Ingold and Yu. V. Nazarov, in: Single Charge Tunneling, ed. by A. Grabert and M. Devoret (Plenum, New York, 1992), Chap. 2.

[29] A. O. Caldeira and A. J. Leggett, Phys. Rev. Lett. 46, 211 (1981); Ann. Phys. (N.Y.) 149, 374 (1983); 153, 445(E) (1984). 
[30] In real experiments, $Z(\omega)$ has frequency dependence and usually it decays at high frequency regime. However, we take Ohmic environment for illustration because Ohmic environment is simpler to treat and illustrates essential features of real experiments.

[31] H. B. Callen and T. A. Welton, Phys. Rev. 83, 34 (1951).

[32] S. R. E. Yang, Solid State Comm. 81, 375 (1992).

[33] The shunt resistor gives only higher order effect and it can be ignored safely in the leading order calculation of the excess noise.

[34] S. Chakravarty, Phys. Rev. Lett. 49, 681 (1982).

[35] M. Reznikov, M. Heiblum, H. Shtrikman, and D. Mahalu, preprint.

[36] R. Brown and E. Šimánek, Phys. Rev. B. 34, 2957 (1986). 


\section{FIGURES}

FIG. 1. (a) Schematic diagram of the circuit. The circuit contains a single tunnel junction , voltage source, and the impedance $Z(\omega)$ of the environment. (b) The tunneling current and the relaxed current. The tunneling current represents the electron flow right at the potential barrier of a junction. As an example, the tunneling current through the insulating barrier in metal-insulator-metal junctions is drawn. The relaxed current represents the electron flow through the environment which is connected to a junction in series. In the figure, Ohmic environment with resistance $R$ is drawn.

FIG. 2. Zero temperature current-voltage characteristics with $Z(\omega)=0.01 R_{Q}$ (solid line), $Z(\omega)=100 R_{Q}($ dashed line $)$, and $Z(\omega)=R_{Q}($ dash-dotted line $)$.

FIG. 3. Zero temperature equilibrium noise spectrum of the tunneling current for $Z(\omega)=0.01 R_{Q}($ solid line $), Z(\omega)=100 R_{Q}($ dashed line $)$, and $Z(\omega)=R_{Q}($ dash-dotted line $)$.

FIG. 4. (a) Zero temperature excess noise spectra of the tunneling current for $Z(\omega)=0.01 R_{Q}($ solid line $), Z(\omega)=100 R_{Q}($ dashed line $)$ and $Z(\omega)=R_{Q}($ dash-dotted line $)$. Voltage bias of $e V=0.5 E_{C}$ is assumed. (b) Evolution of zero temperature tunneling excess noise with voltage bias in the high impedance environment $Z(\omega)=100 R_{Q} \cdot e V=0.5 E_{C}$ (solid line), $e V=1.5 E_{C}($ dashed line $)$, and $e V=2.5 E_{C}$ (dash-dotted line).

FIG. 5. The excess noise spectrum of the relaxed current $S_{I_{R}}^{X}(\omega)$ (solid line) vs. the excess noise spectrum of the tunneling current $S_{I_{T}}^{X}(\omega)$ (dashed line) at zero temperature. The dash-dotted line shows $1 /\left(1+\omega^{2} R^{2} C^{2}\right) S_{I_{T}}^{X}(\omega)$ for comparison. (a) $Z(\omega)=0.01 R_{Q}, e V=200 E_{C}$. The dash-dotted line is not visible because it overlaps with the solid line. (b) $Z(\omega)=100 R_{Q}, e V=0.5 E_{C}$. The solid line and the dash-dotted line are multiplied by $10^{4}$ to magnify their features. Note that $S_{I_{R}}^{X}(\omega)$ is negative. (c) $Z(\omega)=R_{Q}, e V=0.5 E_{C} . S_{I_{R}}^{X}(\omega)$ has a single peak at $\omega=0$ compared to

two peaks of $S_{I_{T}}^{X}(\omega)$. $S_{I_{R}}^{X}(\omega)$ deviates from $1 /\left(1+\omega^{2} R^{2} C^{2}\right) S_{I_{T}}^{X}(\omega)$ due to nontrivial behavior of the zero point fluctuations. 


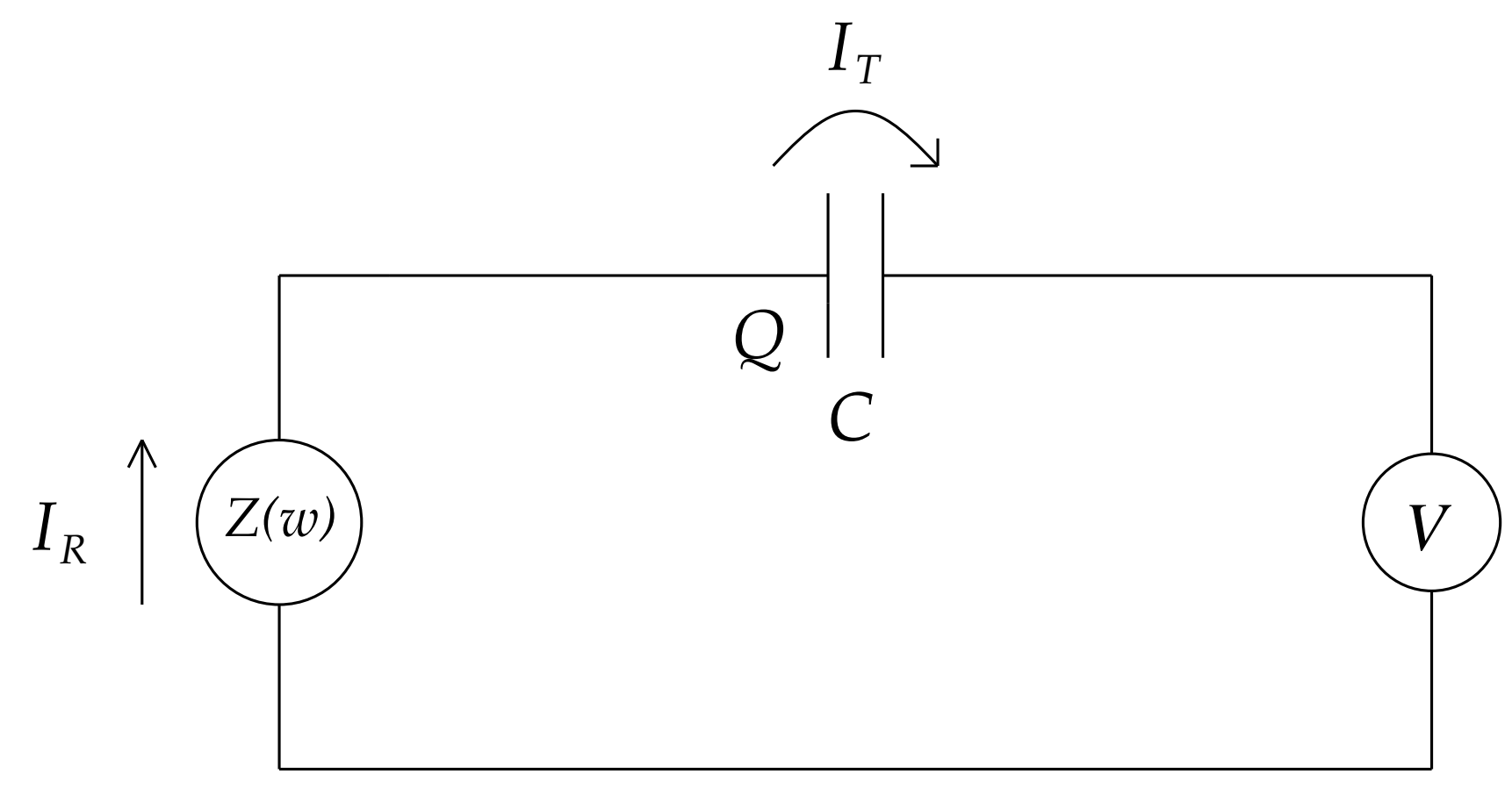




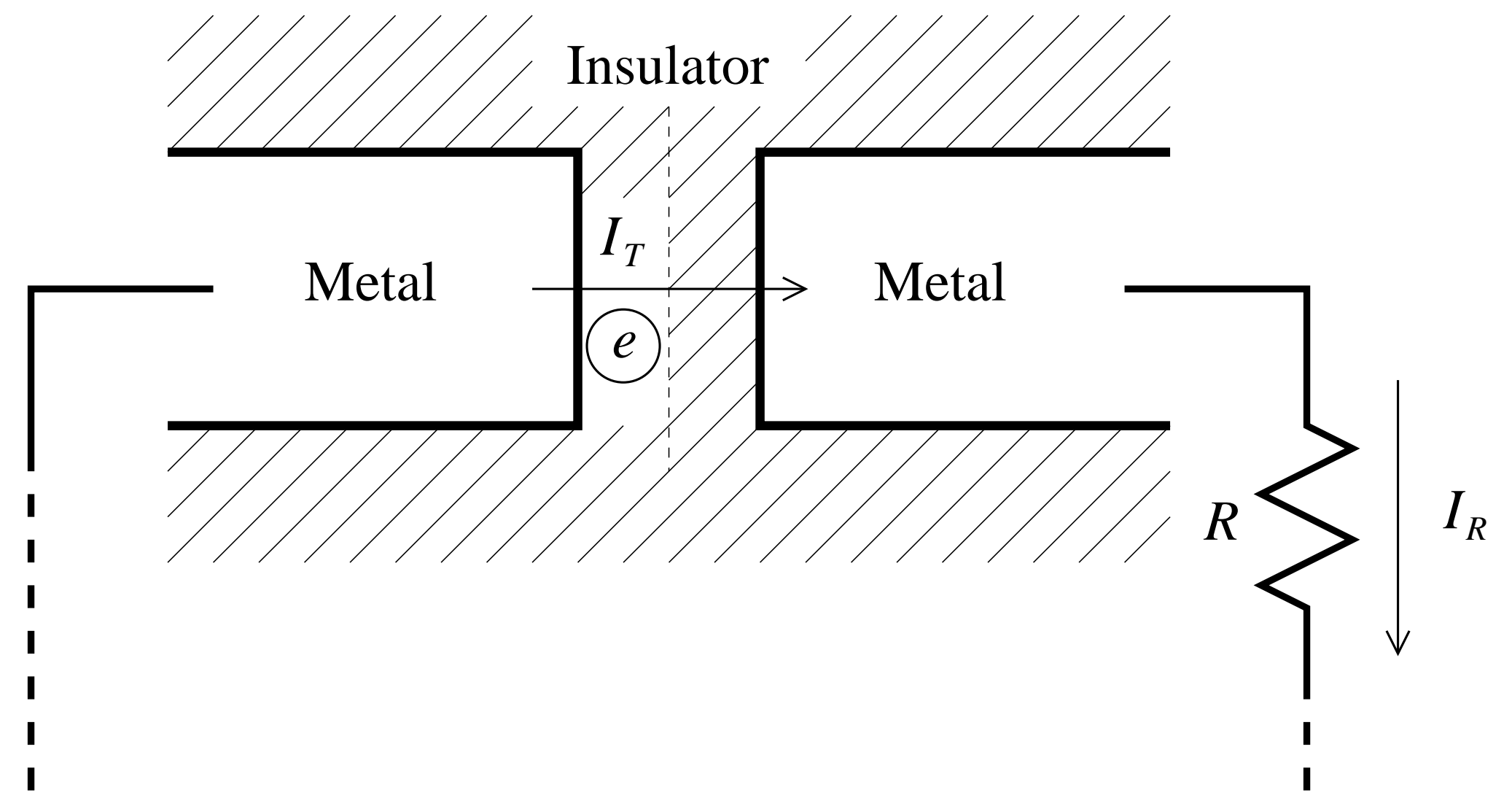




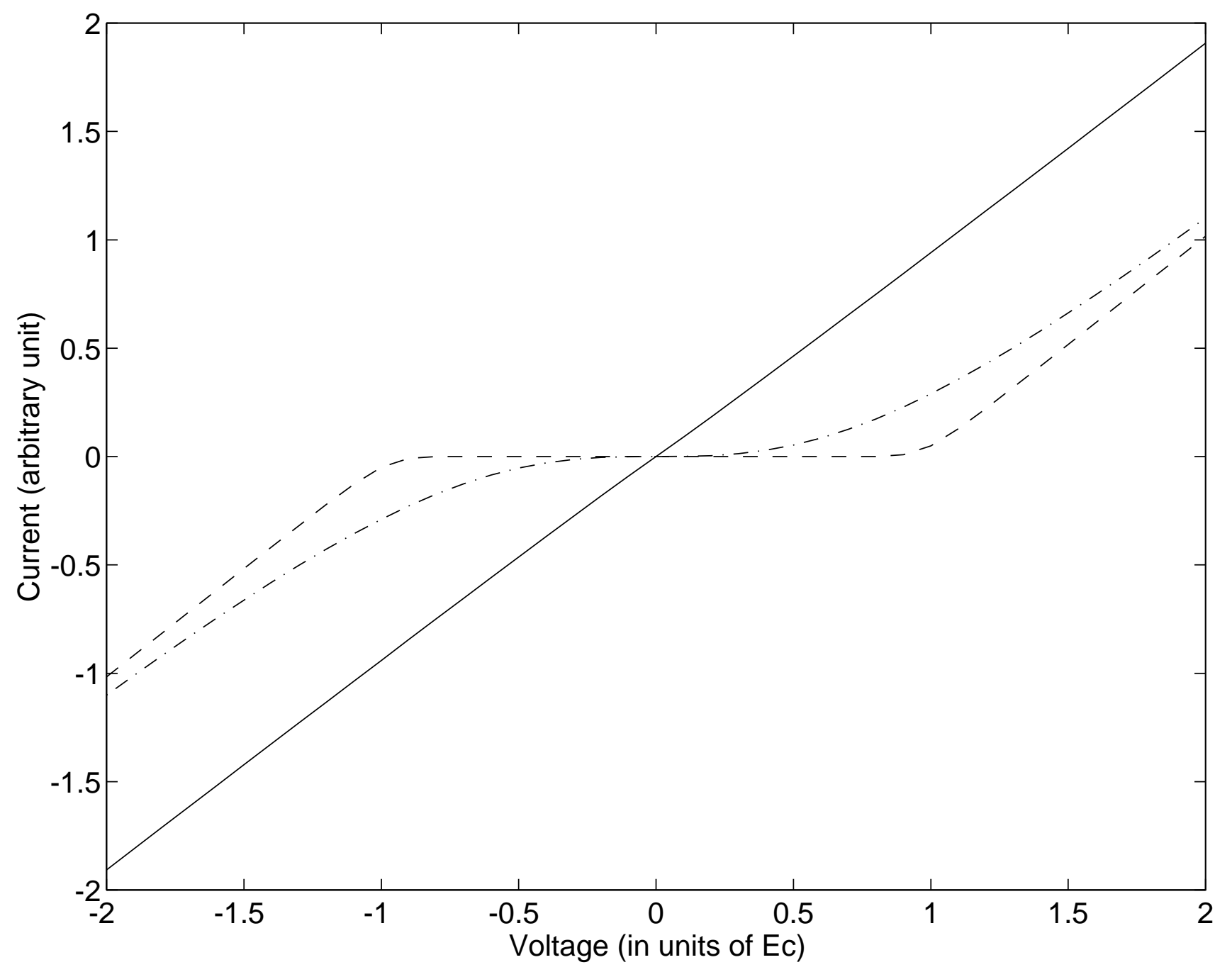




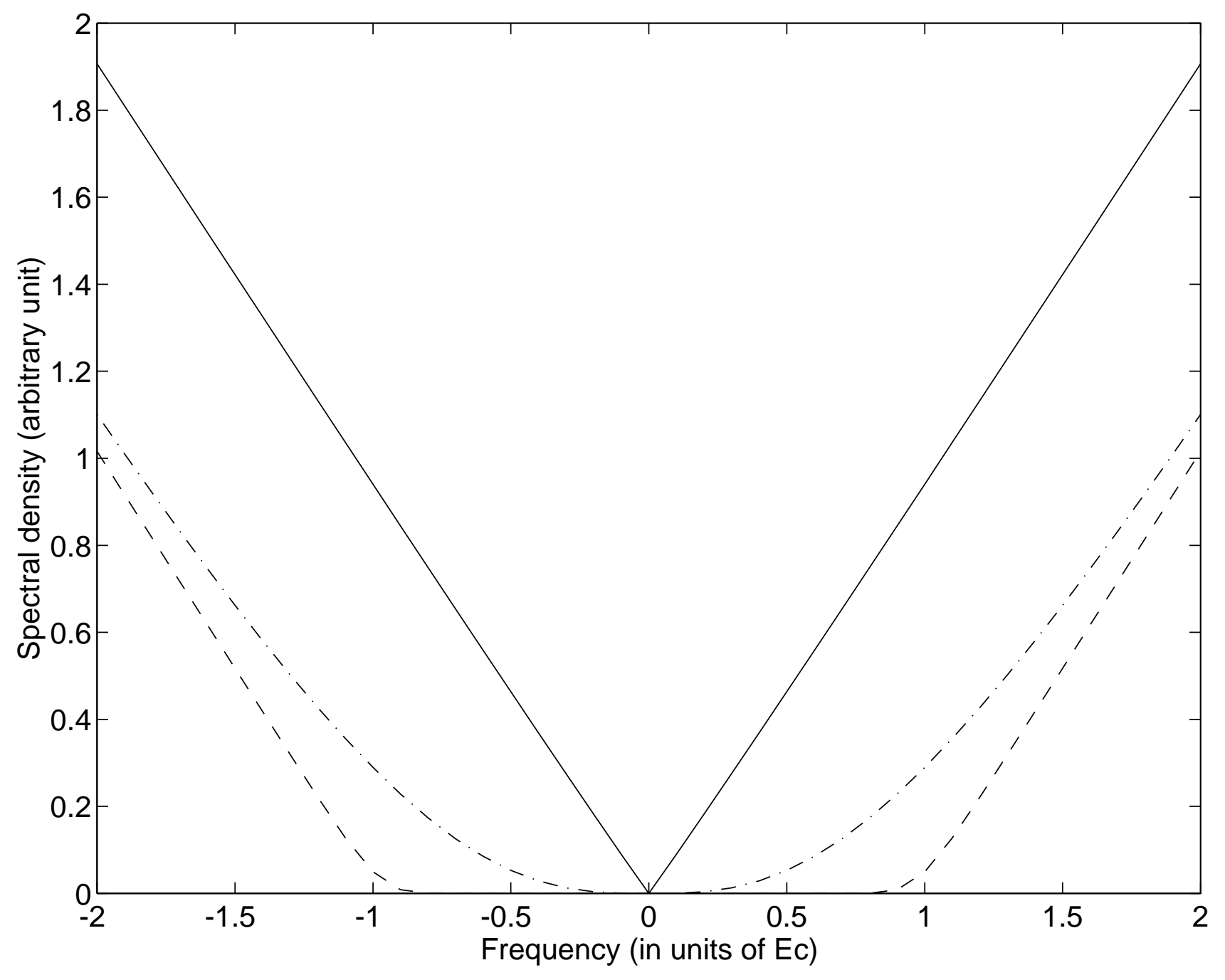




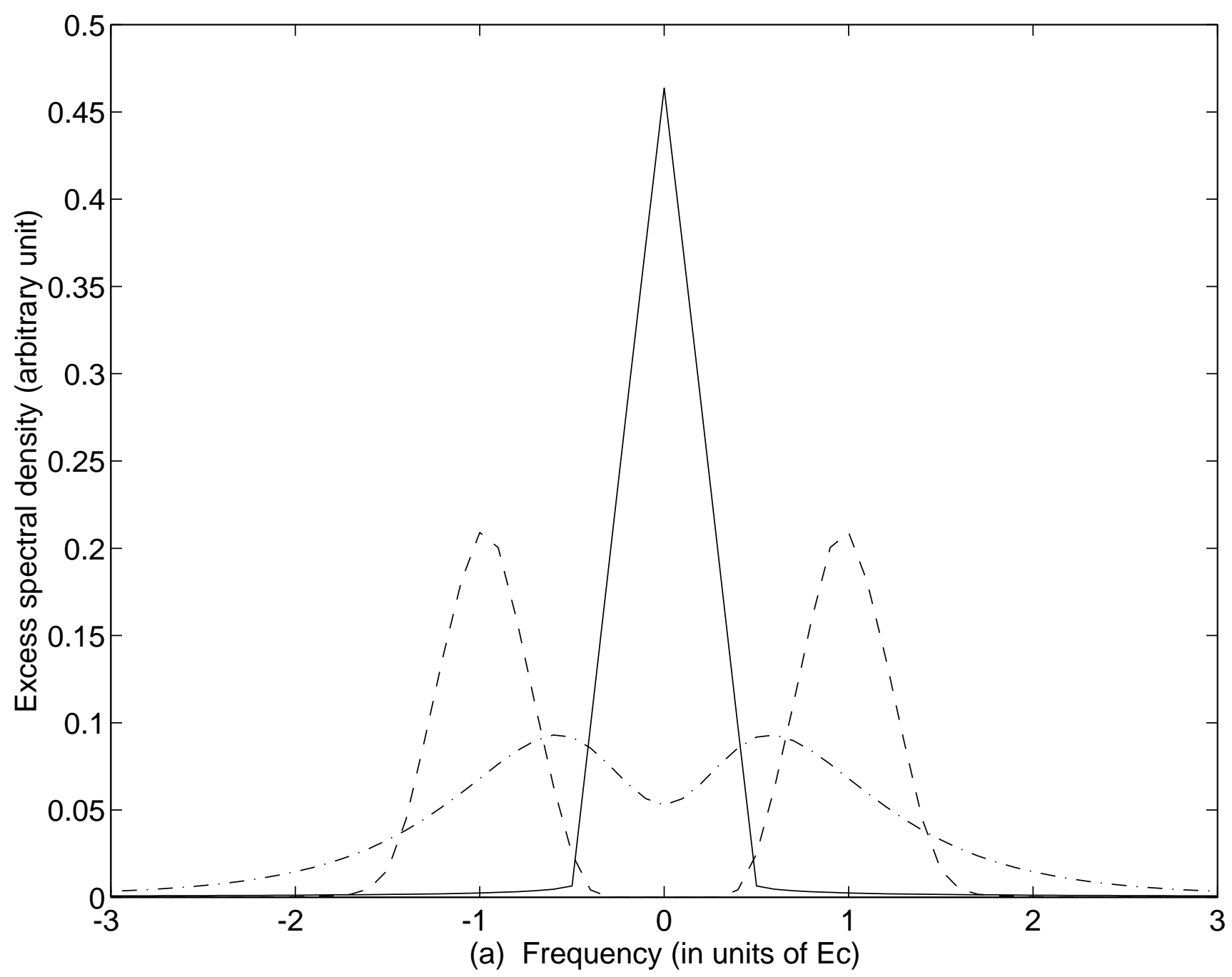




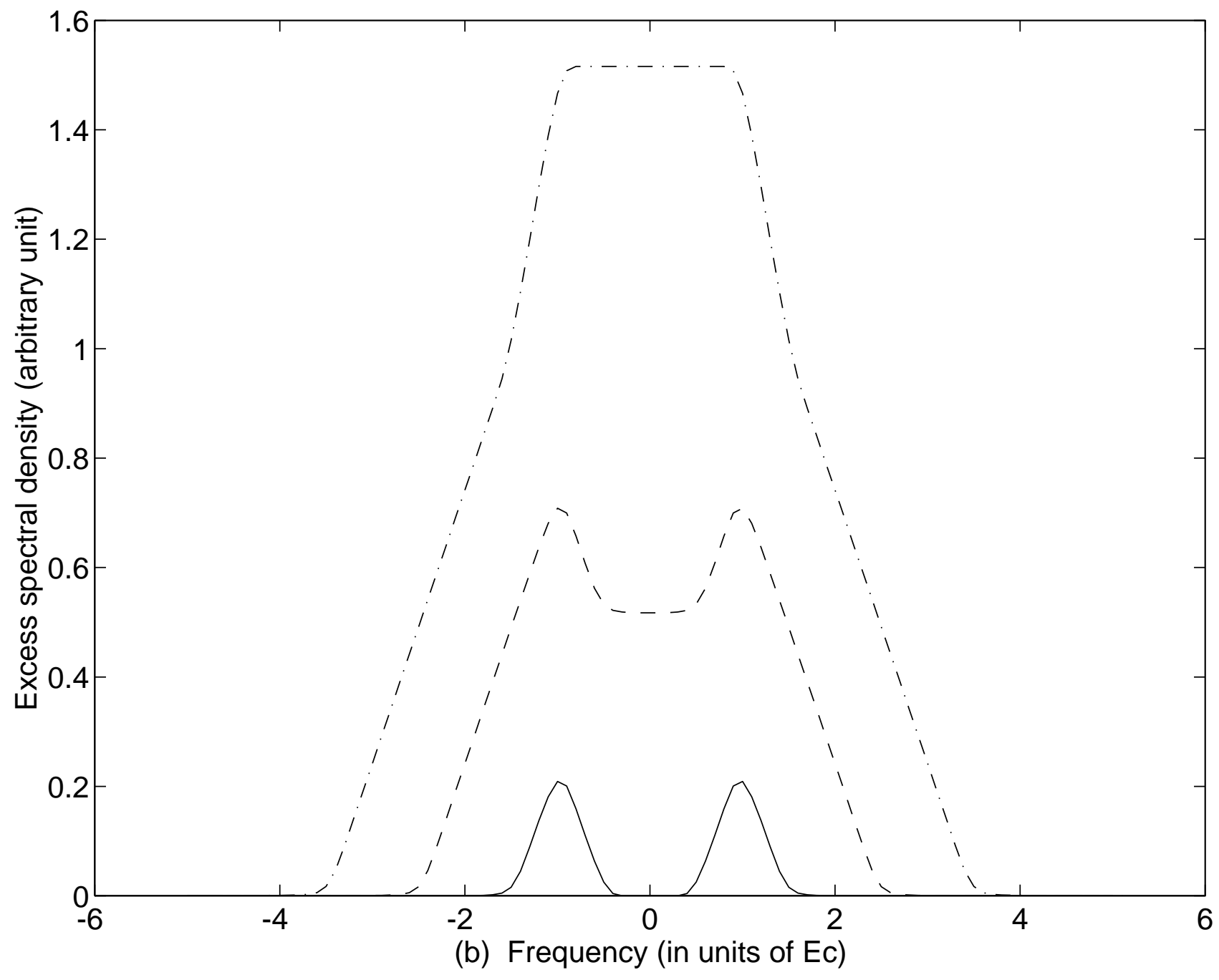




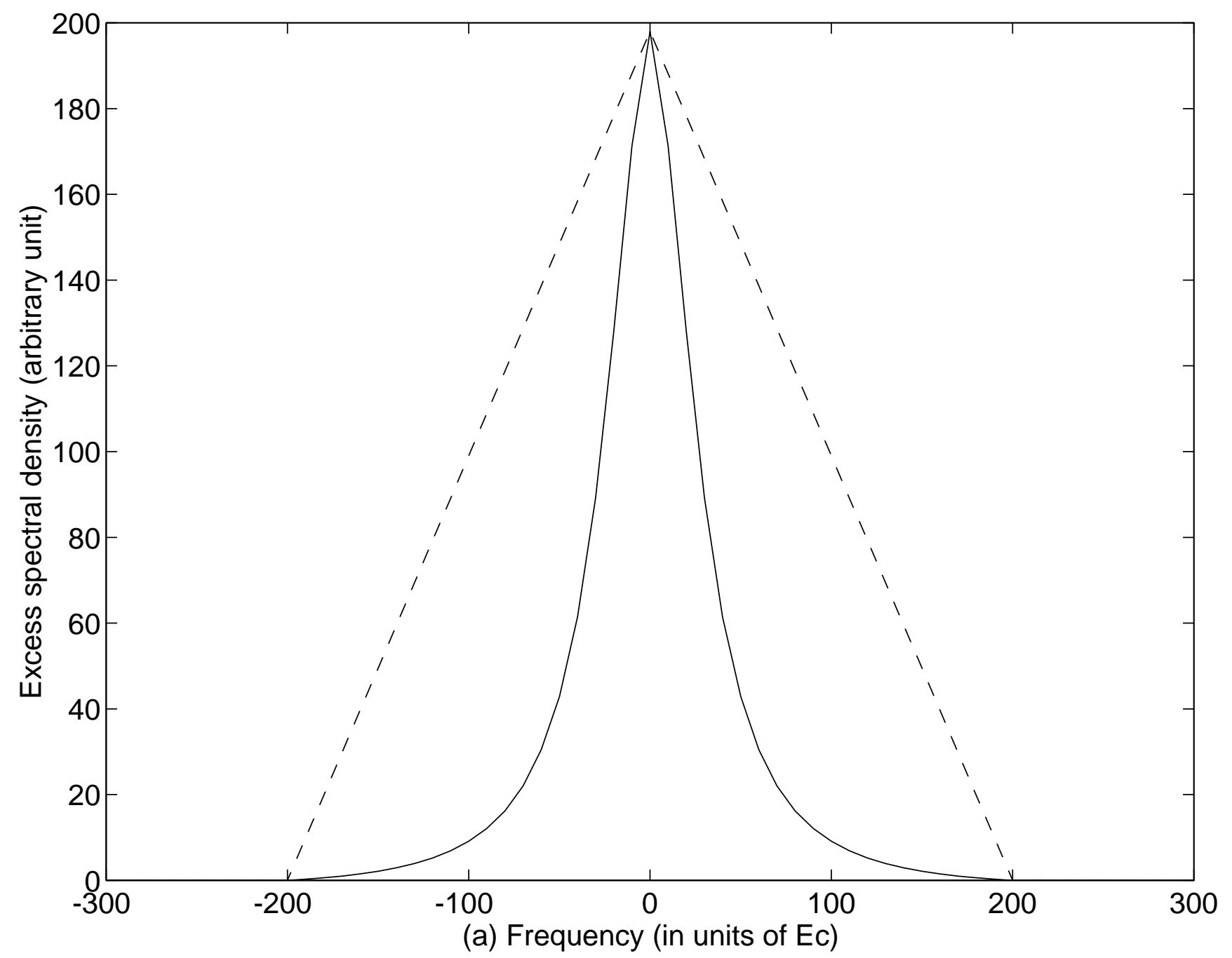




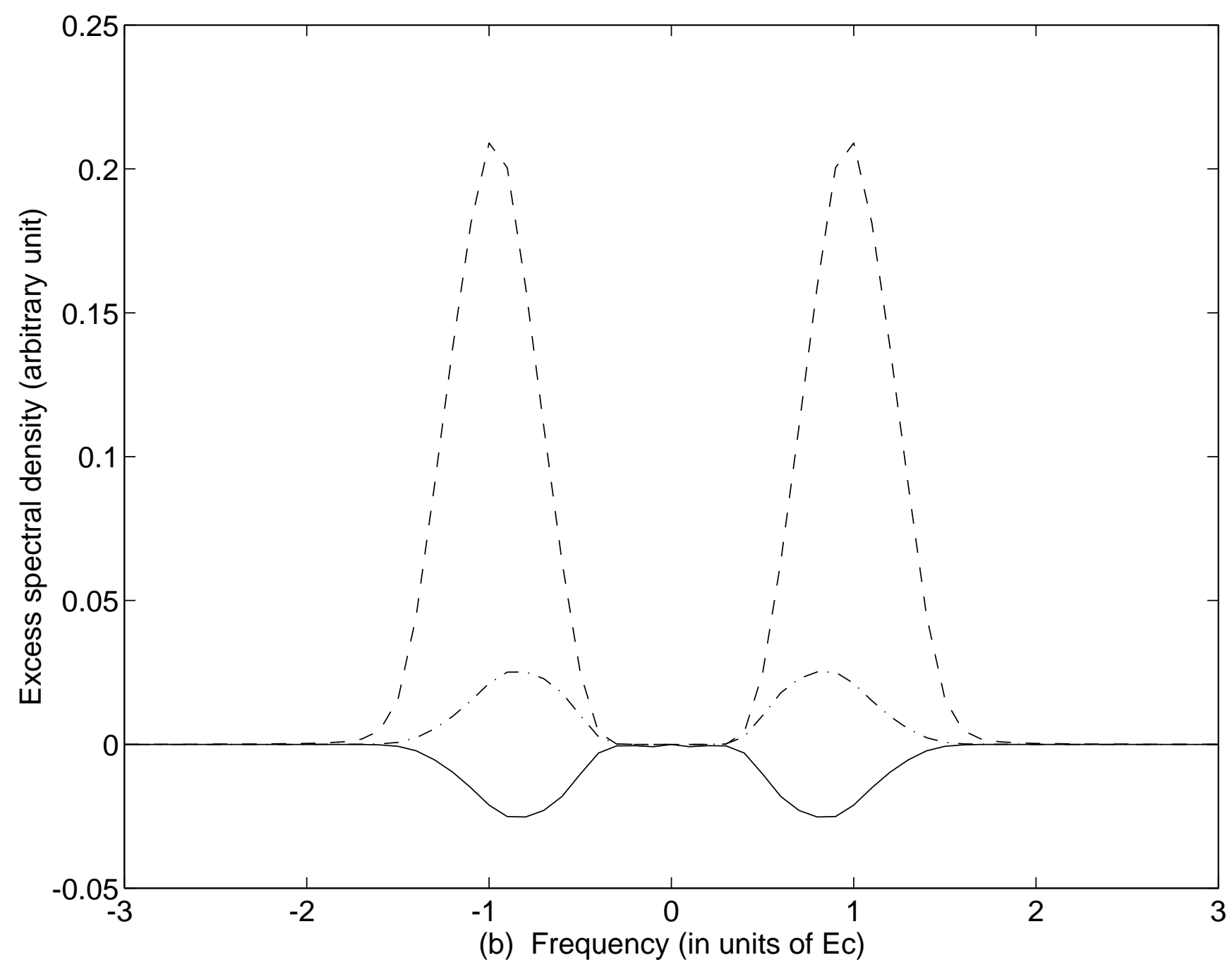




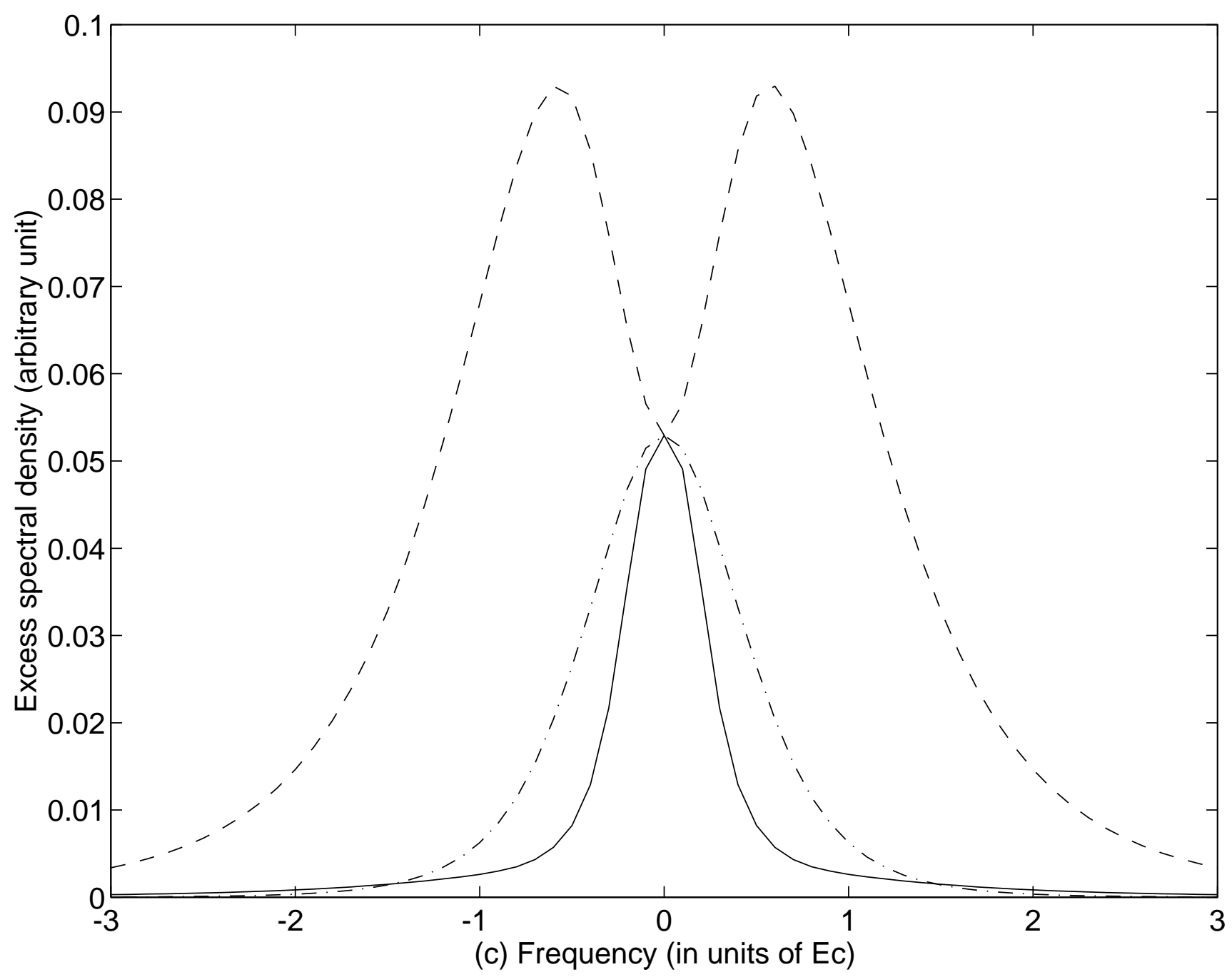

